\title{
Risk assessment of labial bone perforation in the anterior mandibular region: a virtual immediate implant placement study
}

Yi-Wen Cathy Tsai ${ }^{1}$, Ren-Yeong Huang ${ }^{1}$, Chia-Dan Cheng ${ }^{1}$, Wan-Chien Cheng ${ }^{1}$, David L. Cochran², Thomas T. Nguyen ${ }^{3}$, Yi-Shing Shieh ${ }^{4}$, Fu-Gong Lin ${ }^{5,6}$ and Cheng-En Sung ${ }^{1 *}$ (D)

\begin{abstract}
Background: This study investigated the prevalence of labial bone perforation (LBP) related to the associated anatomic factors in anterior mandibular region using a virtual immediate implant placement procedure.

Methods: Series qualified CBCT images of 149 participants (894 teeth) were selected to analyze the assigned anatomical parameters, including concavity depth, concavity angle, torque, and deep bone thickness. Four classes of crestal and radicular dentoalveolar bone phenotypes (CRDAPs) of mandibular anterior teeth were categorized according to the thickness of dentoalveolar bone at both crestal and radicular zones. Data were adjusted for categorical (gender and CRDAP) and continuous (age, cavity angle, cavity depth, and deep bone thickness) variables using a multivariable logistic regression analysis with generalized estimating equation method.

Results: The overall probability of LBP after virtual implant placement was $21.6 \%$. There is statistically significant higher prevalence of LBP at canine (28.5\%) and CRDAP class II (29.2\%) regions $(p<0.001)$. After adjusting confounding variables, CRDAP class II and class IV regions are more likely to have LBP when compared with CRDAP class I (control) regions $(p<0.01)$. The risk of LBP at canine site is 6.31 times more likely than at the central incisor (control) $(p<0.01)$.
\end{abstract}

Conclusions: Using a virtual immediate implant placement technique, the prevalence of LBP is significantly higher at the mandibular canine site and thin radicular dentoalveolar phenotype in the anterior mandibular region.

Keywords: Cone beam computed tomography, Mandible, Classification, Risk assessment, Dental implantation, Labial bone perforation

\section{Introduction}

Currently, placing an implant immediately into an extraction socket is still believed to be a techniquesensitive procedure, even though high survival and success rates of immediate implant treatment have been reported [1-3]. Surgical complications related to immediate implant placement may compromise the outcome and even result in life-threatening events [4-9]. Therefore, preventive and precautionary risk assessment

\footnotetext{
* Correspondence: peter71130000@hotmail.com

'Department of Periodontology, School of Dentistry, Tri-Service General

Hospital and National Defense Medical Center, Taipei, Taiwan

Full list of author information is available at the end of the article
}

should be taken before implant placement to reduce the probability of surgical-related risks and achieve a satisfactory outcome. The operator should conduct comprehensive preoperation risk assessments and apply thorough knowledge of the surgical zone's anatomical features when deciding the optimal position and angulation of implants [4-10].

With regard to anatomic risks during immediate implant placement, many regions of interest have been studied previously [10-15]. For the posterior mandible region, the existence of lingual concavity, perforation of the lingual cortical plate, and geometric features of inferior alveolar nerve in the mandible limit the immediate 
implant placement procedure $[7,10,12,14-16]$. In the anterior maxilla region, labial bone deficiency, and the particular relationship between teeth and alveolar process may increase the risk of perforation of the facial cortical bone $[10,11,17]$. Consequently, soft tissue recession for the thin labial bone thickness of anterior maxillary teeth potentially compromised the esthetic outcomes $[13,18,19]$. Therefore, adequate knowledge of prosthetically driven treatment plan should be applied to prevent vital tissue damage, such as cortical bone perforation.

Although numerous studies regarding the decreased anatomic risks of immediate implant have been proposed, cases about labial bone perforation (LBP) in anterior mandibular region compromising the implant survival have still been reported, but the assessment of risk of LBP in this region is limited [20,21]. Therefore, the purpose of this study was to investigate the prevalence of crestal and radicular dentoalveolar bone phenotype (CRDAP) and evaluate anatomic factors contributing to the prospect of LBP in the anterior mandibular region using a virtual immediate implant placement in cone beam computed tomography (CBCT) images.

\section{Methods}

\section{Database confidentiality, image acquisition, and} retrieving

In this retrospective observational study, consecutive CBCT images were retrieved and investigated from a CBCT database possessed by the Department of Dentistry, Tri-Service General Hospital, National Defense Medical Center, Taipei, Taiwan (from November 2013 to December 2016). All images were not taken specifically for this project. The qualified images (subjects and teeth) that met the inclusion and exclusion criteria were selected for analysis (Supplemental Figure 1 and Supplemental Table 1). The protocol used in this study was reviewed and approved by the Ethics Committee and Institutional Review Board of Tri-Service General Hospital, National Defense Medical Center (TSGHIRB No. 2-10205-064).

The CBCT machine (NewTom 5G; QR, Verona, Italy) was operated by board-certified radiologist following the standard manufacturer's settings as previously described $[10,12]$. The skull orientation and region of interest were taken according to previous studies $[10,15,22]$. The maxilla was bilaterally symmetric and the occlusal plane, either in the frontal or sagittal view, was parallel to the ground (Supplemental Figure 2A). The acquired CBCT images were saved in a Digital Imaging and Communications in Medicine (DICOM) format, and these data were confidentially protected.
Inclusion and exclusion criteria of selected CBCT images The CBCT images had to fulfill the following inclusion and exclusion criteria as previously described [10, 23]. The inclusion criteria had to be as follows [10, 23]:

- Permanent mandibular central incisor, lateral incisor, or canine had to be fully erupted with fully formed apexes;

- Each examined tooth had to be normally positioned with normal alignment, with a harmonious incisal line across the mandibular anterior teeth;

- Opposing maxillary teeth had to be present to provide information for optimal implant angulation and inclination;

Subjects were excluded if images showed [10, 23]:

- Bone screws and plates for surgical treatments, or any grafted materials;

- Preexisting alveolar bone destruction, perforation, dehiscence, or a combination of these caused by periodontal disease or traumatic injury around the investigated region;

- Supernumerary or impacted tooth;

- A pathological lesion, or evident root resorption;

- Incompletely formed apex;

- Dental misalignment, or preexisting dental implant;

- Signs of prosthodontic treatment, root canal treatments, and/or apical surgery;

- Obscurity or distortion due to scattering, or beamhardening artifact reasons.

Assessment and classification of the crestal and radicular dentoalveolar phenotype (CRDAP) of anterior mandibular teeth

The following reference points and landmarks were defined on reconstructed images by viewing a sagittalsectioned image of the region of interest and the center section of each investigated tooth (Fig. 1a) [24].

- Point A and Point B: the mid-facial and mid-lingual CEJ of the tooth, respectively;

- Point $\mathrm{C}$ : the intersection point of line A-B and line connecting the incisal edge and root apex;

- Length of the root (L): the distance between root apex and the Point C;

- Measuring point 1 (MP1): the point located $4 \mathrm{~mm}$ apical to the CEJ on facial root surface;

- Measuring point 2 (MP2): the point located middle of the root on buccal root surface.

Two dentoalveolar zones, "crestal zone" and "radicular zone", were defined with minor modification to fit the purpose of this study accordingly (Fig. 1b) [25]. The crestal zone of dentoalveolar bone was defined as the region from the facial CEJ extending to a point 4-mm apical (MP1). The radicular zone was dependent upon individual root length and was defined as the region from MP1 to the MP2 (Fig. 1). The thickness of facial 


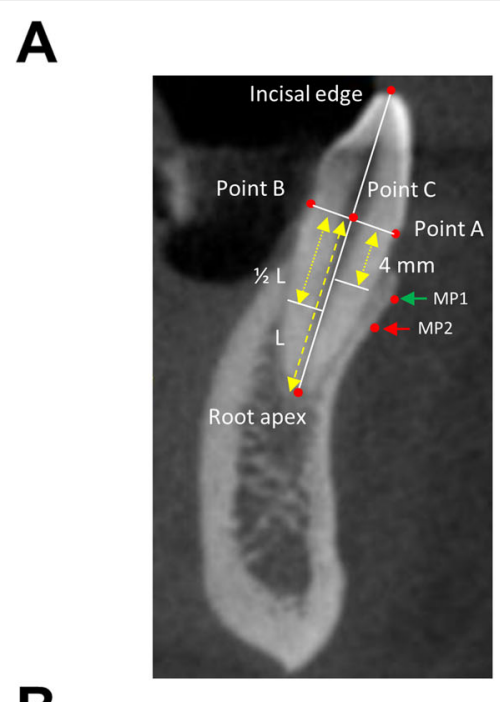

B
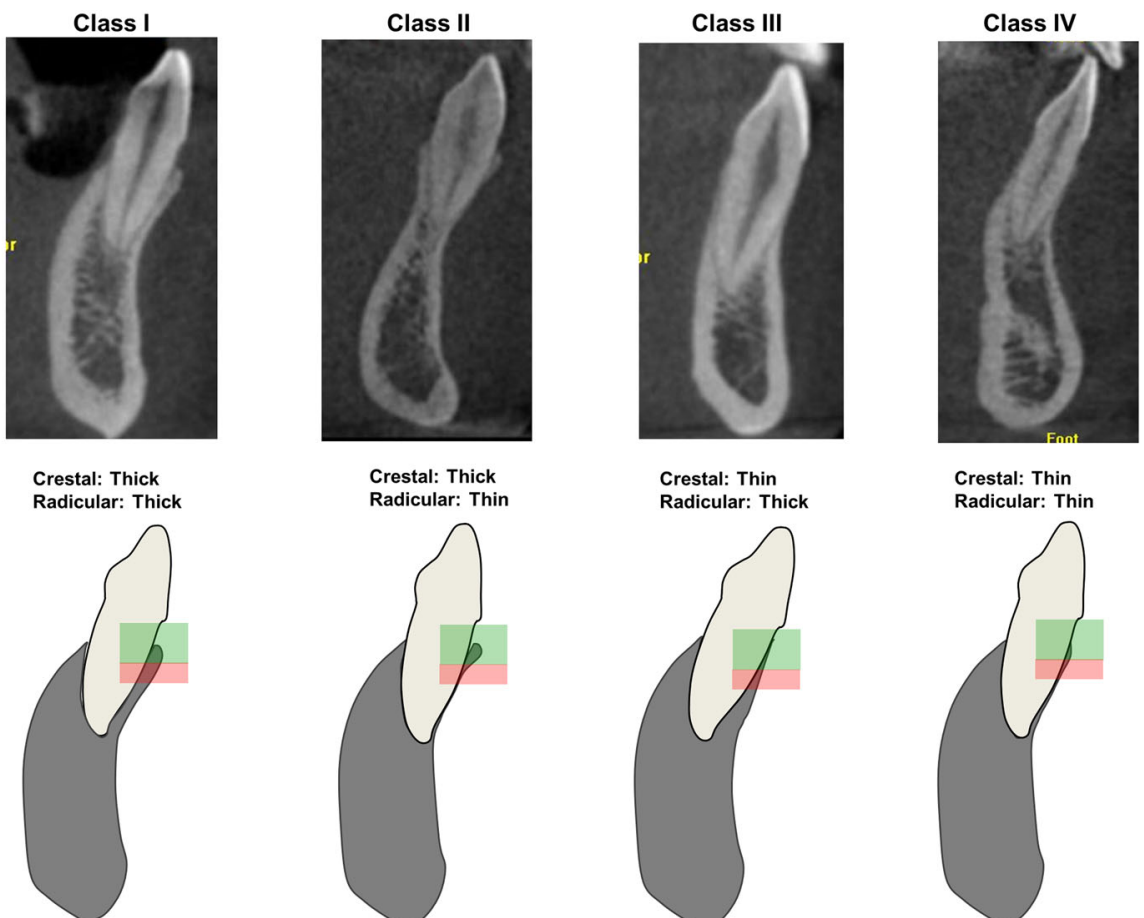

Crestal: Thick Radicular: Thin

Crestal: Thin Radicular: Thick

Crestal: Thin Radicular: Thin
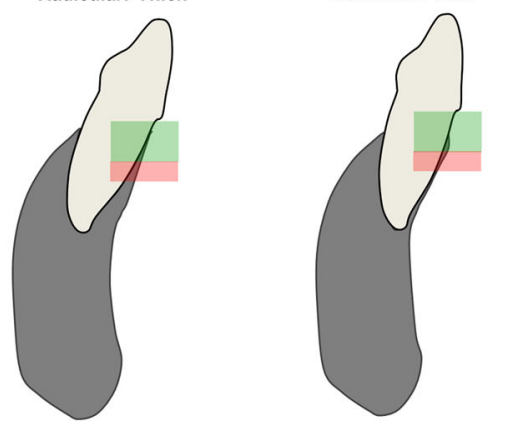

Fig. 1 Landmarks and classification of the crestal and radicular dentoalveolar phenotype (CRDAP) of mandibular anterior teeth in relation to the anterior mandibular osseous housing in sagittal radiographic images. a Landmarks in the sagittal radiographic images. b Radiographic images of four types of CRDAP (upper panel). Schematic illustrations of four types of CRDAP (class I, II, III, and IV) (lower panel), which categorized by the alveolar bone thickness at crestal (green color) and radicular (red color) region

dentoalveolar bone on both crestal and radicular zones could be determined as either thick or thin phenotype [25]. For thick phenotypes, the facial bone thickness was defined as $\geq 1 \mathrm{~mm}$, whereas, for thin phenotypes, the thickness was $<1 \mathrm{~mm}$.

Four classes of crestal and radicular dentoalveolar phenotype (CRDAP) of mandibular anterior teeth were categorized according to the thickness of dentoalveolar bone at both crestal and radicular zones at the tooth level (Fig. 1b) [25]:

- Class I: both the crestal and radicular dentoalveolar zones were thick phenotype.

- Class II: the crestal zone was thick, but the radicular zone was thin phenotype

- Class III: the radicular zone was thick, but the crestal zone was thin phenotype 
- Class IV: both the crestal and radicular dentoalveolar zones were thin

phenotype.

\section{Measurements of morphological features of mandibular anterior region}

The following morphologic and dimensional parameters of the mandibular teeth and alveolar ridge were measured accordingly (Fig. 2) [10, 17, 26].

- Concavity depth (CD): the distance between the deepest point of the facial bone plate (point Q) and a vertical reference line perpendicular to the mandibular plane, passing through the most external point of the labial plate (point P) (Fig. 2a).

- Concavity angle (CA): the angulation between line Q-P (that is, the line connecting points $\mathrm{Q}$ and $\mathrm{P}$, with point $\mathrm{P}$ defined as the most external point of the labial plate) and line Q-R (that is, the line connecting points $Q$ and $\mathrm{R}$, with point $\mathrm{R}$ defined as the most external point of the labial plate inferior to point $Q$, and relatively lower than the apex of the tooth along the apico-coronal continuum) (Fig. 2a).

- Torque (T): the angle formed between the long axis of a tooth (that is, the line connecting incisal edge and root apex of the tooth) and long axis of the mandible, connecting from root apex to point $\mathrm{D}$ (that is, the lowest point of the mandible) (Fig. 2b) [26].
- Deep bone thickness (dBT): mandibular deep bone thickness was measured at 0,5 , and $10 \mathrm{~mm}$ from tooth root apex and along the long axis of the mandible (that is, the line connecting the tooth apex and point D), presented as $\mathrm{dBT}(0), \mathrm{dBT}(5)$, and $\mathrm{dBT}$ (10), respectively (Fig. 2b). Each measurement line was also perpendicular to the mandibular long axis, then the distance between the facial and lingual outline of the bone was measured [26].

\section{Virtual implant selection, placement, and definition of labial bone perforation (LBP)}

The root form taper-designed dental implants were selected from an implant database available in the CBCT software as previously described $[10,12,16]$. The diameter of dental implants was determined by mimicking the corresponding size of each investigated tooth root, $3.0 \mathrm{~mm}$ for central and lateral incisors and $4.3 \mathrm{~mm}$ for canines (NobelActiveTM, Nobel Biocare, Gothenburg, Sweden) respectively, which were commonly used values for anterior mandibular teeth [27].

A selected dental implant was virtually placed along the long axis of the investigated tooth root with $4 \mathrm{~mm}$ of implant anchorage into native bone that was considered the minimum necessary to achieve primary stability [28, 29]. After placing the suitable implant virtually, labial bone perforation (LBP) or non-perforation were
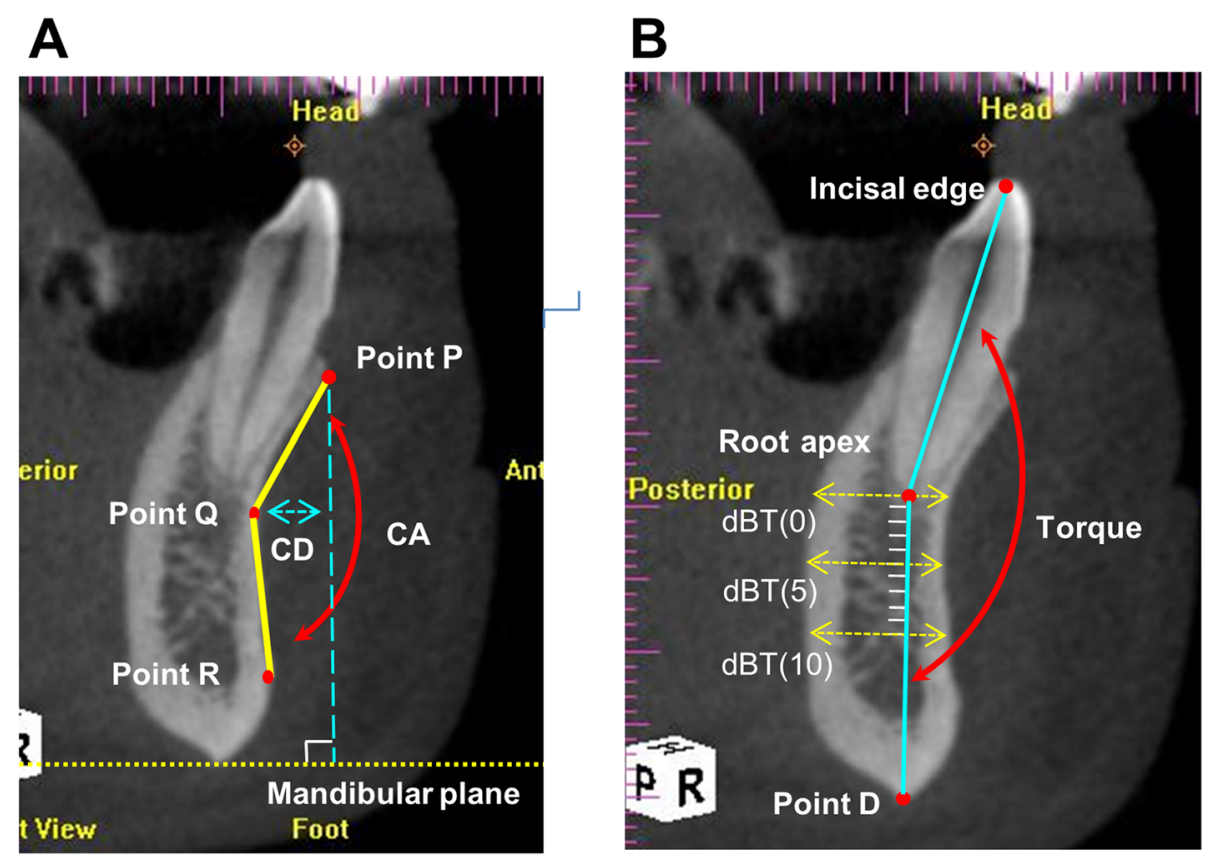

Fig. 2 Anatomical parameters regarding labial bone perforation (LBP) of the anterior mandibular region in sagittal radiographic images. a Landmarks and anatomical parameters of the concavity depth (CD), and concavity angle (CA). $\mathbf{b}$ Landmarks and anatomical parameters of the torque (T), and deep bone thickness (dBT). Each measurement line of dBT was also perpendicular to the mandibular long axis, and then the distance between the facial and lingual outline of the bone was measured 
determined. The LBP was defined when the virtual implant extruded out of the apical outline of the labial cortical bone in the cross-sectioned and axial-viewed images (Supplemental Figure 3A) [14]. On the contrary, non-perforation was defined as the virtual implant within the apical outline of the labial cortical bone (Supplemental Figure 3B).

\section{Qualification and examination of $\mathrm{CBCT}$ images}

All selected CBCT images of $1920 \times 1080$ pixel resolution, displayed on a 19-inch liquid-crystal display monitor (ChiMei Innolux Corporation, Taiwan), were examined by a commercially available threedimensional (3D) navigation software (ImplantMax ${ }^{\circ}$ 4.0; Saturn Image, Taipei, Taiwan) in a dimly lit environment. To ensure data reliability and reproducibility, all images were re-oriented so that the morphology of the crown and root, from cementoenamel junction (CEJ) to the apex, in the sagittal planes could be investigated undoubtedly as described previously (Supplemental Figure 2B) [22].

\section{Calibration and reliability between intra- and inter- examiners}

The CBCT images were carefully inspected to follow the eligibility criteria by two independent investigators (first and corresponding author) twice, 1 week apart. Prior to the study, intra- and inter-examiner calibrations were performed on 50 randomly selective images based on the diagnosis of anatomic landmarks and anatomical measurements from $\mathrm{CBCT}$ images to assess data reliability. The nominal variables (e.g., perforation vs. nonperforation, CRDAP classification, Supplemental Table 2) and continuous variables (e.g., concavity depth, torque, $\mathrm{dBT}(0, \mathrm{~mm}), \mathrm{dBT}(5, \mathrm{~mm}), \mathrm{dBT}(10, \mathrm{~mm}))$ and calibrated measurement errors, Cronbach's alpha, and ICC (intraclass correlation coefficient) between intra- and interobservations were also summarized (Supplemental Table 3). The Kappa statistic values for CRDAP and labial bone perforation were 0.940 and 0.929 for intra-observer agreement, and 0.929 and 0.932 for inter-observer agreement, respectively (Supplemental Table 2). Furthermore, the measurement errors, intraclass correlation coefficient, and Cronbach $\alpha$ values were also performed to confirm the reliability of intra- and inter-observations for continuous variable measurement (Supplemental Table 3). After calibration, the two independent investigators (first and corresponding author) evaluated the images separately, and any disagreement in image interpretation was discussed until a consensus was reached.

\section{Statistical analysis}

The occurrences of LBP were expressed as the percentage of the number of sites with perforation divided by the total number of corresponding investigated sites. Pearson's chi-square tests were used to examine differences with categorical variables, such as the frequency distribution of four types of CRDAP classification and the LBP of the investigated tooth in the anterior mandibular zone. Shapiro-Wilk test was used for testing the normality of data (data not shown). To compare the values of $\mathrm{CD}, \mathrm{CA}, \mathrm{T}$, and deep bone thickness at perforation and non-perforation sites, Mann-Whitney $U$ tests were performed for the non-normality. After multicollinearity tests were performed to examine the linearity between each two variables, dBT (5) was removed from final regression analysis (data not shown). For analyzing the risk of LBP, multivariable logistic regression analysis with generalized estimating equation (GEE) method was used to handle repeated measurements of tooth sites in each subject which simultaneously adjusted for within factors of person and tooth sites, categorical (i.e., gender, tooth type, and crestal and radicular dentoalveolar phenotype (CRDAP)) and continuous (i.e., age, cavity depth (CD), cavity angle (CA), torque $(\mathrm{T})$, and deep bone thickness $(\mathrm{dBT})$ ) variables. Four models were applied where Model 1 was adjusted for within factors (as our univariate model), Model 2 was adjusted for within factors, gender, and age, Model 3 was adjusted for within factors, gender, age, CD, CA, $\mathrm{T}, \mathrm{dBT}(0)$, and $\mathrm{dBT}(10)$, and Model 4 was adjusted for within factors, gender, age, $\mathrm{CA}, \mathrm{CD}, \mathrm{T}, \mathrm{dBT}(0), \mathrm{dBT}$ (10), and CRDAP (CRDAP class III teeth were excluded due to no "perforation" teeth in this group). These adjusted variables in sequential models were determined to follow a theme from within factors, subject's factors, and then anatomic factors influencing labial bone perforation with evidences proven by previous studies. All statistical analyses were performed by SPSS for Windows (PASW Statistics, version 18.0, SPSS, Inc., Chicago, IL, USA) and the level of statistical significance was set at $p<0.05$.

\section{Results \\ Qualified participants and CRDAP distribution}

A total of 149 participants (894 teeth) retrieved from database met the inclusion criteria. The frequency distribution of CRDAP classification of investigated teeth was class IV $(60.0 \%)$, followed by class II $(18.8 \%)$, class I (10.9\%), and class III (10.4\%) (Table 1). Among the CRDAP class IV, canine teeth $(70.5 \%)$ had the highest prevalence, compared with lateral incisors (56.4\%) and central incisors (53.0\%) (Table 1$)$.

\section{Frequency distribution of labial bone perforation (LBP)}

The overall probability of LBP of all investigated teeth was $21.6 \%$ (193 teeth), and the LBP was most likely to occur in canines (85 teeth; $28.5 \%$ ) when compared with central and lateral incisors (Table 2). When comparing 
Table 1 Frequency distribution of four types of crestal and radicular dentoalveolar phenotype (CRDAP) of investigated teeth in the anterior mandibular region

\begin{tabular}{|c|c|c|c|c|c|c|c|c|c|c|}
\hline \multirow[b]{2}{*}{ Variables } & \multirow{2}{*}{$\begin{array}{l}\text { Total } \\
n\end{array}$} & \multicolumn{2}{|c|}{ CRDAP Class I } & \multicolumn{2}{|c|}{ CRDAP Class II } & \multicolumn{2}{|c|}{ CRDAP Class III } & \multicolumn{2}{|c|}{ CRDAP Class IV } & \multirow[b]{2}{*}{$p$} \\
\hline & & $n$ & $\%$ & $n$ & $\%$ & $n$ & $\%$ & $n$ & $\%$ & \\
\hline Side & & & & & & & & & & 0.487 \\
\hline Right & 447 & 44 & 9.8 & 92 & 20.6 & 46 & 10.3 & 265 & 59.3 & \\
\hline Left & 447 & 53 & 11.9 & 76 & 17.0 & 47 & 10.5 & 271 & 60.6 & \\
\hline Tooth type & & & & & & & & & & $<0.001$ \\
\hline Central incisor & 298 & 58 & 19.5 & 56 & 18.8 & 26 & 8.7 & 158 & 53.0 & \\
\hline Lateral incisor & 298 & 25 & 8.4 & 74 & 24.8 & 31 & 10.4 & 168 & 56.4 & \\
\hline Canine & 298 & 14 & 4.7 & 38 & 12.8 & 36 & 12.1 & 210 & 70.5 & \\
\hline Side and tooth type & & & & & & & & & & $<0.001$ \\
\hline Central incisor, right & 149 & 24 & 16.1 & 31 & 20.8 & 13 & 8.7 & 81 & 54.4 & \\
\hline Central incisor, left & 149 & 34 & 22.8 & 25 & 16.8 & 13 & 8.7 & 77 & 51.7 & \\
\hline Lateral incisor, right & 149 & 13 & 8.7 & 38 & 25.5 & 16 & 10.7 & 82 & 55.0 & \\
\hline Lateral incisor, left & 149 & 12 & 8.1 & 36 & 24.2 & 15 & 10.1 & 86 & 57.7 & \\
\hline Canine, right & 149 & 7 & 4.7 & 23 & 15.4 & 17 & 11.4 & 102 & 68.5 & \\
\hline Canine, left & 149 & 7 & 4.7 & 15 & 10.1 & 19 & 12.8 & 108 & 72.5 & \\
\hline Total & 894 & 97 & 10.9 & 168 & 18.8 & 93 & 10.4 & 536 & 60.0 & \\
\hline
\end{tabular}

The classification of crestal and radicular dentoalveolar phenotype (CRDAP) of mandibular anterior teeth was categorized according to the thickness of dentoalveolar bone at both crestal and radicular zones [25]. The chi-square tests were used for comparing the distribution of CRDAP in different "side," "tooth type," and "side and tooth type," respectively. The level of statistical significance was set at $p<0.05$

the risk of LBP using the CRDAP classification, class II had the highest probability of LBP (29.2\%) compared with other CRDAP classes (Table 2). The significant differences were observed between tooth type $(p<0.001)$ and CRDPA classification $(p<0.001)$ (Table 2).

\section{Morphologic parameters related to LBP}

The concavity depth (CD) and torque (T) in the perforation group were statistically higher than in the nonperforation group, whereas concavity angle (CA) was lower in the perforation group than in the nonperforation group (Table 3). All these values had a significant impact on the probability of LBP (all $p<0.001$ ). The bone thickness increased gradually toward the apical region, from dBTs (0) $(8.20 \pm 2.02 \mathrm{~mm}), \mathrm{dBTs}(5)$ $(9.41 \pm 2.04 \mathrm{~mm})$, to dBTs $(10)(10.84 \pm 2.00 \mathrm{~mm})$, but bone thickness was statistically lower in perforation group than non-perforation group at each measured level $(p<0.001)$ (Table 3).

\section{Further evaluation with regression model, including tooth-, alveolar ridge-, and tooth-alveolar ridge- associated factors}

A multivariable logistic regression analysis with generalized estimating equation method was used which simultaneously adjusted for categorical and continuous variables as previously described (Table 4). Placing virtually placed immediate implants at the canines were 6.31 times more likely to cause LBP when compared with the central incisors (reference group) $(P<0.01)$ (Table 4$)$. The deep bone thickness at $0 \mathrm{~mm}(\mathrm{dBT}(0))$ significantly influenced the probability of LBP $(P<0.01)$, but not at $10 \mathrm{~mm}$. The results also showed that when the tooth is classified as CRDAP class II or class IV, it is much more likely to have LBP when compared with the CRDAP class I (reference group), respectively (Table 4).

\section{Discussion}

This research evaluated the anatomic phenotype of the anterior mandible using the CRDAP classification and demonstrated a high prevalence of thin labial bone plate. In addition, the alveolar morphologic structure, including concavity depth (CD), concavity angle (CA), and deep bone thickness ( $\mathrm{dBT})$, might increase the risk of labial bone plate perforation and complicate the outcome in immediate implant placement (IIP) over anterior mandibular region. Therefore, this study highlights the importance of preoperative evaluation using 3dimensional CBCT images.

In this study, the CRDAP class IV (60.0\%) was the most prevalent in the anterior mandible, indicating the facial bone wall in the crestal and radicular area of teeth were thin (Table 1). This is in line with previous studies which showed that in the majority of the examined teeth, a thin facial bone pattern $(\leq 1 \mathrm{~mm})$ is exhibited at different levels $[24,30]$, which may imply the high prevalence of CRDAP class IV (Table 1). Consequently, thin facial bone wall may require simultaneous bone 
Table 2 Frequency distribution of labial bone perforation of investigated teeth in the anterior mandibular region

\begin{tabular}{|c|c|c|c|c|c|}
\hline \multirow[b]{2}{*}{ Variables } & \multicolumn{2}{|c|}{ Perforation } & \multicolumn{2}{|c|}{ Non-perforation } & \multirow[b]{2}{*}{$p$} \\
\hline & $n$ & $\%$ & $n$ & $\%$ & \\
\hline Total & 193 & 21.6 & 701 & 78.4 & \\
\hline Side & & & & & 0.464 \\
\hline Right & 101 & 22.6 & 346 & 77.4 & \\
\hline Left & 92 & 20.6 & 355 & 79.4 & \\
\hline Tooth type & & & & & $<0.001$ \\
\hline Central incisor & 63 & 21.1 & 235 & 78.9 & \\
\hline Lateral incisor & 45 & 15.1 & 253 & 84.9 & \\
\hline Canine & 85 & 28.5 & 213 & 71.5 & \\
\hline Side and tooth type & & & & & 0.002 \\
\hline Central incisor, right & 38 & 25.5 & 111 & 74.5 & \\
\hline Central incisor, left & 25 & 16.8 & 124 & 83.2 & \\
\hline Lateral incisor, right & 21 & 14.1 & 128 & 85.9 & \\
\hline Lateral incisor, left & 24 & 16.1 & 125 & 83.9 & \\
\hline Canine, right & 42 & 28.2 & 107 & 71.8 & \\
\hline Canine, left & 43 & 28.9 & 106 & 71.1 & \\
\hline Tooth-alveolar ridge (CRDAP) & & & & & $<0.001^{*}$ \\
\hline CRDAP class I & 3 & 3.1 & 94 & 96.9 & \\
\hline CRDAP class $\|$ & 49 & 29.2 & 119 & 70.8 & \\
\hline CRDAP class III & 0 & 0.0 & 93 & 100.0 & \\
\hline CRDAP class IV & 141 & 26.3 & 395 & 73.7 & \\
\hline
\end{tabular}

The classification of crestal and radicular dentoalveolar phenotype (CRDAP) of mandibular anterior teeth was categorized according to the thickness of dentoalveolar bone at both crestal and radicular zones [25]. The "perforation" was defined as the virtual implant extruded out of the apical outline of the labial cortical bone, whereas "non-perforation" was defined as the virtual implant within outline of the labial cortical bone. The chi-square tests were used for examining the frequency distribution of labial bone perforation ("perforation" vs. "non-perforation") in different "sides," "tooth types," and "side and tooth types," respectively. *The Fisher's exact test was used to examine the frequency distribution of labial bone perforation ("perforation" vs. "non-perforation") in different "tooth-alveolar ridge (CRDAPs)." The level of statistical significance was set at $p<0.05$

augmentation procedures during immediate implant placement because substantial bone resorption following tooth extraction occurs at a thin facial bone thickness $[31,32]$. This is also the first study to investigate the prevalence of CRDAP classifications in different tooth types, in which canines account for the majority at CRDAP class IV (70.5\%, Table 1$)$.

In regard to the rate of $\mathrm{LBP}$, the risk of perforation is closely related to tooth type $(p<0.001)$ and CRDAP classification $(p<0.001)$ (Table 2$)$. The canine site (28.5\%) presented highest prevalence of LBP, followed by central incisor (21.1\%), and lateral incisor (15.1\%). In terms of CRDAP classification, similar prevalence of LBP occurs in CRDAP class II (29.2\%) and class IV (26.3\%) teeth. In a previous study using navigated flapless transmucosal approach to place inter-foraminal implant in edentulous mandibles, two implants (2.5\%), both of canines, had to be removed immediately due to buccal bone perforation [20], which corresponds with our present results that LBP occurred most frequently at canine sites compared to other anterior mandibular teeth (Table 2). Although immediate implant placement has had a high cumulative success rate in the anterior mandible region, significant dimensional changes of the facial bone are to be expected after replacing a tooth with a dental implant [21]. Therefore, thorough evaluation of a potential implant site, including preoperative assessment of thin facial bone wall (i.e., canine or CRDAP class IV), can indicate sites which may require bone augmentation or guided bone regeneration procedures to allow for reconstruction of the hard tissue deficiency and adequate support for esthetically pleasing soft tissues [24, 33].

The information about these anatomic features contributing to the occurrence of LBP in the anterior mandible region is limited. In this study, the regional anatomical parameters, including $\mathrm{CD}, \mathrm{CA}, \mathrm{T}$, and $\mathrm{dBT}$ (Table 3), significantly influence the occurrence of LBP, compared with the non-perforation sites. Moreover, perforation was 6.31 more likely to occur at canine sites than central incisors (reference group), and toothalveolar ridge relationship classified as CRDAP class II or class IV was much more likely to have LBP when compared with CRDAP class I (reference group) (Table 4). Thus, the specific tooth type and tooth-alveolar ridge relationship could have a significant impact on the occurrence of LBP (all $p<0.01$, Table 4). Taken together, it is necessary to take these anatomical factors into consideration while performing comprehensive pre-surgical examination.

Clinically, the ideal immediate implant position is based on a restoration-driven treatment plan with precise 3-D positioning of the implant, rather than on the amount of available bone [34]. According to the results of the current study, the probability of LBP in anterior mandibular region is common $(21.6 \%$, Table 2 ). Therefore, based on favorable long-term outcome, the simultaneous guided bone regeneration or delayed implant placement should be considered when the implant sites were associated with inadequate bone volume or quality [31, 32].

The prevailing concept for pre-surgical treatment plan should consist of precise 3-D positioning of the implant and guided implant surgery for facilitating a more predictable treatment outcome, and improve patient safety [35, 36]. Specifically, by reducing probability of LBP, surgeons are highly suggested to perform meticulous preoperative examinations and continuously re-evaluate the decision using 3-D CBCT analysis before placing dental implant into extraction placement immediately $[26,35,36]$. 
Table 3 Comparisons of concavity depth (CD, mm), concavity angle (CA, degree), torque (degree), and deep bone thickness (dBT, $\mathrm{mm})$ at different levels $(0,5$, and $10 \mathrm{~mm})$ in the "perforation" and "non-perforation" sites of investigated teeth in anterior mandibular region

\begin{tabular}{|c|c|c|c|c|c|c|c|}
\hline \multirow[b]{2}{*}{ Variables } & \multicolumn{2}{|l|}{ Total } & \multicolumn{2}{|l|}{ Perforation } & \multicolumn{2}{|c|}{ Non-perforation } & \multirow[b]{2}{*}{$p^{*}$} \\
\hline & Mean (SD) & Median (IQR) & Mean (SD) & Median (IQR) & Mean (SD) & Median (IQR) & \\
\hline \multicolumn{8}{|l|}{ Alveolar ridge } \\
\hline Concavity depth (CD) & $4.44(1.96)$ & $4.2(3.0)$ & $5.59(1.93)$ & $5.6(2.9)$ & $4.12(1.85)$ & $3.8(2.6)$ & $<0.001$ \\
\hline Cavity angle (CA) & $144.95(7.36)$ & $145.4(9.4)$ & $140.53(7.13)$ & $142.1(9.0)$ & $146.17(6.95)$ & $146.7(8.8)$ & $<0.001$ \\
\hline \multicolumn{8}{|l|}{ Deep bone thickness } \\
\hline $\mathrm{dBT}(0)$ & $8.20(2.02)$ & $8.0(2.7)$ & $6.87(1.67)$ & $6.6(2.6)$ & $8.57(1.95)$ & $8.4(2.8)$ & $<0.001$ \\
\hline $\mathrm{dBT}(5)$ & $9.41(2.04)$ & $9.3(2.6)$ & $8.01(1.79)$ & $8.0(2.8)$ & $9.80(1.93)$ & $9.7(2.6)$ & $<0.001$ \\
\hline $\mathrm{dBT}(10)$ & $10.84(2.00)$ & $10.8(2.5)$ & $9.77(1.96)$ & $10.0(2.0)$ & $11.14(1.90)$ & $11.0(2.6)$ & $<0.001$ \\
\hline \multicolumn{8}{|l|}{ Tooth-alveolar ridge } \\
\hline Torque (T) & $161.40(8.62)$ & $161.9(12.1)$ & $166.29(7.94)$ & $167.4(11.3)$ & $160.05(8.32)$ & $160.8(10.9)$ & $<0.001$ \\
\hline
\end{tabular}

The deep bone thickness (dBT) was measured at 0,5 , and $10 \mathrm{~mm}$ from tooth root apex and along the long axis of the mandible, presented as dBT (0), dBT (5), and dBT (10), respectively [26]. The "perforation" was defined as the virtual implant extruded out of the apical outline of the labial cortical bone, whereas "nonperforation" was defined as the virtual implant within outline of the labial cortical bone. *The Mann-Whitney $U$ tests were used to examine the differences of $C D$, $\mathrm{CA}, \mathrm{T}$, and $\mathrm{dBT}$ at "perforation" and "non-perforation" sites. The level of statistical significance was set at $p<0.05$

Abbreviation: IQR interquartile range, $S D$ standard deviation

Table 4 Multiple logistic regression analysis of variables contributing to labial bone perforation during virtual implant placement

\begin{tabular}{|c|c|c|c|c|c|c|c|c|}
\hline \multirow[b]{2}{*}{ Variables } & \multicolumn{2}{|c|}{ Model $1(n=894)$} & \multicolumn{2}{|c|}{ Model $2(n=894)$} & \multicolumn{2}{|c|}{ Model $3(n=894)$} & \multicolumn{2}{|c|}{ Model $4(n=801)$} \\
\hline & $\mathrm{OR}^{*}{ }^{* *}$ & $95 \% \mathrm{Cl}$ & Adjusted OR & $95 \% \mathrm{Cl}$ & Adjusted OR & $95 \% \mathrm{Cl}$ & Adjusted OR & $95 \% \mathrm{Cl}$ \\
\hline \multicolumn{9}{|l|}{ Tooth } \\
\hline Central incisor & Referent & & Referent & & Referent & & Referent & \\
\hline Lateral incisor & $0.66^{* *}$ & $0.49-0.90$ & $0.66^{* *}$ & $0.48-0.90$ & $0.41^{* *}$ & $0.24-0.73$ & $0.29^{* *}$ & $0.16-0.53$ \\
\hline Canine & $1.49^{*}$ & $1.08-2.06$ & $1.50^{*}$ & $1.08-2.08$ & $11.67^{* *}$ & $5.27-25.85$ & $6.31^{* *}$ & $2.85-13.97$ \\
\hline \multicolumn{9}{|l|}{ Alveolar ridge } \\
\hline Concavity depth (CD) & $1.47^{* *}$ & $1.27-1.70$ & $1.49^{* *}$ & $1.29-1.72$ & $1.56^{* *}$ & $1.26-1.94$ & $1.85^{* *}$ & $1.47-2.32$ \\
\hline Cavity angle (CA) & $0.90^{* *}$ & $0.87-0.93$ & $0.89^{* *}$ & $0.86-0.92$ & $0.85^{* *}$ & $0.80-0.90$ & $0.84^{* *}$ & $0.79-0.89$ \\
\hline \multicolumn{9}{|l|}{ Deep bone thickness } \\
\hline $\mathrm{dBT}(0)$ & $0.59^{* *}$ & $0.51-0.67$ & $0.58^{* *}$ & $0.50-0.66$ & $0.43^{* *}$ & $0.34-0.55$ & $0.46^{* *}$ & $0.36-0.58$ \\
\hline $\mathrm{dBT}(10)$ & $0.68^{* *}$ & $0.59-0.78$ & $0.68^{* *}$ & $0.59-0.77$ & 1.03 & $0.84-1.28$ & 1.03 & $0.81-1.31$ \\
\hline \multicolumn{9}{|l|}{ Tooth-alveolar ridge } \\
\hline Torque $(\mathrm{T})$ & $1.10^{* *}$ & $1.06-1.14$ & $1.10^{* *}$ & $1.07-1.14$ & $1.25^{* *}$ & $1.18-1.34$ & $1.28^{* *}$ & $1.21-1.36$ \\
\hline \multicolumn{9}{|l|}{ CRDAP classification } \\
\hline CRDAP class I & - & - & - & - & - & - & Referent & \\
\hline CRDAP class II & - & - & - & - & - & - & $21.39^{* *}$ & $3.04-150.23$ \\
\hline CRDAP class III & - & - & - & - & - & - & - & - \\
\hline CRDAP class IV & - & - & - & - & - & - & $47.26^{* *}$ & $7.00-319.22$ \\
\hline
\end{tabular}

The classification of crestal and radicular dentoalveolar phenotype (CRDAP) of mandibular anterior teeth was categorized according to the thickness of dentoalveolar bone at both crestal and radicular zones [25]. A multivariable logistic regression analysis with generalized estimating equation (GEEs) method was used and which was simultaneously adjusted for categorical (i.e., gender, tooth types, and crestal and radicular dentoalveolar phenotype (CRDAP)) and continuous (i.e., age, cavity depth $(\mathrm{CD})$, cavity angle $(\mathrm{CA})$, torque $(\mathrm{T})$, and deep bone thickness (dBT) variables. * and ${ }^{*}$ indicate statistical differences as $p<0.05, p<0.01$ Model 1: adjusted for within factors

Model 2: adjusted for within factors, gender and age

Model 3: adjusted for within factors, gender, age, tooth types, CD, CA, T, dBT (0), and dBT (10)

Model 4: adjusted for within factors, gender, age, tooth types, CD, CA, T, dBT (0), dBT (10), and CRDAP (CRDAP class III teeth were excluded due to no "perforation" teeth in this group).

Abbreviation: $O R$ odds ratio, 95\% Cl 95\% confidence interval 
There are certain concerns when applying the findings of this study to clinical scenarios. First, computer-guided implant planning has proven to improve the predictability of the treatment goals and risk management [35]; however, further evaluations regarding the accuracy in transferring the virtual designed implant position intraorally are still needed. Second, to prevent the occurrence of LBP, the long axis and position of implant may be adjusted or shifted lingually in clinical setting, which may incidentally damage the lingual arteries in anterior mandibular region, causing massive hemorrhage and even life-threatening events $[5,8]$. Moreover, with the limitation of study design, the subjects' general medical information (diabetes, osteoporosis, arthritis, immune status, etc.) and the cause of tooth loss could not be evaluated. In this study, subjects with severe alveolar destruction or obvious periodontal destruction were excluded to minimize the possibilities of subject-related factors [37, 38]. Concerning the measurement errors from CBCT, previous studies showed the mean absolute errors between $\mathrm{CBCT}$ and direct measurements appear to be limited and are unlikely to be clinically relevant with proper preliminary calibration [39]. The images with poorer quality, which resulted from minor patient movements during imaging which might contribute to measurement discrepancy between the image dimensions and realistic morphologies, were also excluded [40]. The current results regarding the prevalence of associated anatomical factors (i.e., CRDAP, $\mathrm{CA}, \mathrm{CD}, \mathrm{T}, \mathrm{dBT}$ ) show significant contributions to the occurrence of LBP while placing immediate implant in anterior mandible region. These results would provide further referent information, which constructs a preoperative automatic knowledgebased algorithm in CBCT image to assess risk in immediate implant surgery with identified important anatomic factors.

\section{Conclusion}

To reduce the probability of LBP, meticulous preoperative assessments on anatomical parameters are highly suggested when placing dental implant immediately in the anterior mandible region.

\begin{abstract}
Abbreviations
CRDAP: Crestal and radicular dentoalveolar bone phenotype; CBCT: Cone beam computed tomography; DICOM: Digital imaging and communications in medicine; LBP: Labial bone perforation; CD: Concavity depth; CA: Concavity angle; T: Torque; dBT: Deep bone thickness; GEE: Generalized estimating equation
\end{abstract}

\section{Supplementary Information}

The online version contains supplementary material available at https://doi. org/10.1186/s40729-021-00351-w.

Additional file 1: Supplemental Figure 1 (A) The flowchart of subject and image recruitment protocol. Subjects for full edentulous ridge, dental misalignment, alveolar bone destruction, and distorted image were excluded. (B) Images for tooth missing or implant replacement, pathologic lesion, and prosthodontic treatment were excluded. Supplemental Figure 2 (A) A schematic description of the image orientation procedure. (B) The virtual implant position was verified mesiodistally (upper panel) and buccolingually (lower left panel) from reconstructed 3-dimensional images (lower right panel). Supplemental Figure 3 Definition of labial bone perforation (LBP) in the anterior mandibular region while performing virtual immediate implant placement. (A) The perforation was defined when the virtual implant extruded out of the outline of the labial cortical bone in the cross-sectioned and axialviewed images. (B) The non-perforation was defined as the virtual implant within the apical outline of the labial cortical bone.

Additional file 2.

\section{Acknowledgements}

The authors appreciated Ms. Yi-Shing Lin and Ms. Li-Chin Yu (Tri-Service General Hospital) for their help in CT taking and imaging processing. We also thank Ms. Yu-Fang Huang (School of Dentistry, National Defense Medical Center) and Mr. Charles Chapman (Center of General Education, National Defense Medical Center) for their help in manuscript editing.

\section{Authors' contributions}

All authors have made substantial contributions to conception and design of the study. C-ES, Y-WCT, and R-YH, C-DC, W-CC, DLC, TTN, Y-SS, and F-GL have been involved in data collection and data analysis. C-ES, Y-WCT, R-YH, C-DC, W-CC, DLC, TTN, Y-SS, and F-GL have been involved in data interpretation. CES, Y-WCT, R-YH, C-DC, W-CC, DLC, TTN, Y-SS, and F-GL have been involved in drafting the manuscript. C-ES, Y-WCT, R-YH, C-DC, W-CC, DLC, TTN, Y-SS, and F-GL have been involved in revising it critically and have given final approval of the version to be published.

\section{Funding}

The study was kindly supported by Medical Affairs Bureau, Ministry of National Defense, Taiwan (MND-MAB-1 10-052) and Tri-Service General Hospital (TSGH-C108-184, TSGH-D-109169, TSGH-D-110145).

\section{Availability of data and materials}

The datasets of the current study are available from the corresponding author on reasonable request.

\section{Declarations}

Ethics approval and consent to participate

The research protocol was approved by the Ethics Committee and Institutional Review Board of Tri-Service General Hospital, National Defense Medical Center (TSGHIRB No. 2-102-05-064).

Consent for publication

All authors have approved the publication of this manuscript.

\section{Competing interests}

Yi-Wen Cathy Tsai, Ren-Yeong Huang, Chia-Dan Cheng, Wan-Chien Cheng, David L. Cochran, Thomas T. Nguyen, Yi-Shing Shieh, Fu-Gong Lin, and Cheng-En Sung declare that they have no competing interests.

\section{Author details}

${ }^{1}$ Department of Periodontology, School of Dentistry, Tri-Service General Hospital and National Defense Medical Center, Taipei, Taiwan. ${ }^{2}$ Department of Periodontics, The University of Texas Health Science Center at San Antonio, San Antonio, TX, USA. ${ }^{3}$ Division of Periodontics, Faculty of Dentistry, McGill University, Montreal, QC, Canada. ${ }^{4}$ Department of Oral Diagnosis and Pathology, School of Dentistry, Tri-Service General Hospital and National Defense Medical Center, Taipei, Taiwan. ${ }^{5}$ School of Public Health, National Defense Medical Center, Taipei, Taiwan. ${ }^{6}$ Department of Optometry, University of Kang Ning, Taipei, Taiwan. 
Received: 7 March 2021 Accepted: 29 April 2021 Published online: 26 July 2021

\section{References}

1. Chrcanovic BR, Albrektsson T, Wennerberg A. Dental implants inserted in fresh extraction sockets versus healed sites: a systematic review and metaanalysis. J Dent. 2015;43(1):16-41. https://doi.org/10.1016/j.jdent.2014.11.007.

2. Mello CC, Lemos CAA, Verri FR, Dos Santos DM, Goiato MC, Pellizzer EP. Immediate implant placement into fresh extraction sockets versus delayed implants into healed sockets: a systematic review and meta-analysis. Int J Oral Maxillofac Surg. 2017:46(9):1162-77. https://doi.org/10.1016/j.ijom.2017. 03.016.

3. Lang NP, Pun L, Lau KY, Li KY, Wong MC. A systematic review on survival and success rates of implants placed immediately into fresh extraction sockets after at least 1 year. Clin Oral Implants Res. 2012;23(Suppl 5):39-66. https://doi.org/10.1111/j.1600-0501.2011.02372.x.

4. Balaguer-Marti JC, Penarrocha-Oltra D, Balaguer-Martinez J, PenarrochaDiago M. Immediate bleeding complications in dental implants: a systematic review. Med Oral Patol Oral Cir Bucal. 2015;20(2):e231-8.

5. Woo BM, Al-Bustani S, Ueeck BA. Floor of mouth haemorrhage and lifethreatening airway obstruction during immediate implant placement in the anterior mandible. Int J Oral Maxillofac Surg. 2006;35(10):961-4. https://doi. org/10.1016/j.jiom.2006.03.020.

6. Isaacson TJ. Sublingual hematoma formation during immediate placement of mandibular endosseous implants. J Am Dent Assoc. 2004;135(2):168-72. https://doi.org/10.14219/jada.archive.2004.0148.

7. Kalpidis CD, Setayesh RM. Hemorrhaging associated with endosseous implant placement in the anterior mandible: a review of the literature. J Periodontol. 2004;75(5):631-45. https://doi.org/10.1902/jop.2004.75.5.631.

8. Dubois L, de Lange J, Baas E, Van Ingen J. Excessive bleeding in the floor of the mouth after endosseus implant placement: a report of two cases. Int J Oral Maxillofac Surg. 2010;39(4):412-5. https://doi.org/10.1016/j.jom.2009.07. 062.

9. Givol N, Chaushu G, Halamish-Shani T, Taicher S. Emergency tracheostomy following life-threatening hemorrhage in the floor of the mouth during immediate implant placement in the mandibular canine region. J Periodontol. 2000;71(12):1893-5. https://doi.org/10.1902/jop.2000.71.12.1893.

10. Sung CE, Cochran DL, Cheng WC, Mau LP, Huang PH, Fan WH, et al. Preoperative assessment of labial bone perforation for virtual immediate implant surgery in the maxillary esthetic zone: a computer simulation study. J Am Dent Assoc. 2015;146(11):808-19. https://doi.org/10.1016/j.adaj.2015.04. 007.

11. Gluckman H, Pontes CC, Du Toit J. Radial plane tooth position and bone wall dimensions in the anterior maxilla: a CBCT classification for immediate implant placement. J Prosthet Dent. 2018;120(1):50-6. https://doi.org/10.101 6/j.prosdent.2017.09.005.

12. Huang RY, Cochran DL, Cheng WC, Lin MH, Fan WH, Sung CE, et al. Risk of lingual plate perforation for virtual immediate implant placement in the posterior mandible: a computer simulation study. J Am Dent Assoc. 2015; 146(10):735-42. https://doi.org/10.1016/j.adaj.2015.04.027.

13. Cosyn J, Eghbali A, Hermans A, Vervaeke S, De Bruyn H, Cleymaet R. A 5year prospective study on single immediate implants in the aesthetic zone. J Clin Periodontol. 2016;43(8):702-9. https://doi.org/10.1111/jcpe.12571.

14. Chan HL, Benavides E, Yeh CY, Fu JH, Rudek IE, Wang HL. Risk assessment of lingual plate perforation in posterior mandibular region: a virtual implant placement study using cone-beam computed tomography. J Periodontol. 2011;82(1):129-35. https://doi.org/10.1902/jop.2010.100313.

15. Chan HL, Brooks SL, Fu JH, Yeh CY, Rudek I, Wang HL. Cross-sectional analysis of the mandibular lingual concavity using cone beam computed tomography. Clin Oral Implants Res. 2011;22(2):201-6. https://doi.org/1 0.1111/j.1600-0501.2010.02018.x.

16. Lin MH, Mau LP, Cochran DL, Shieh YS, Huang PH, Huang RY. Risk assessment of inferior alveolar nerve injury for immediate implant placement in the posterior mandible: a virtual implant placement study. J Dent. 2014;42(3):263-70. https://doi.org/10.1016/j.jdent.2013.12.014.

17. Chan HL, Garaicoa-Pazmino C, Suarez F, Monje A, Benavides E, Oh TJ, et al. Incidence of implant buccal plate fenestration in the esthetic zone: a cone beam computed tomography study. Int J Oral Maxillofac Implants. 2014; 29(1):171-7. https://doi.org/10.11607/jomi.3397.

18. AlTarawneh S, AlHadidi A, Hamdan AA, Shaqman M, Habib E. Assessment of bone dimensions in the anterior maxilla: a cone beam computed tomography study. J Prosthodont. 2018;27(4):321-8. https://doi.org/10.1111/ jopr.12675.

19. Buser D, Chappuis V, Belser UC, Chen S. Implant placement post extraction in esthetic single tooth sites: when immediate, when early, when late? Periodontol 2000. 2017;73(1):84-102.

20. Wittwer G, Adeyemo WL, Schicho K, Figl M, Enislidis G. Navigated flapless transmucosal implant placement in the mandible: a pilot study in 20 patients. Int J Oral Maxillofac Implants. 2007;22(5):801-7.

21. Covani U, Chiappe G, Bosco M, Orlando B, Quaranta A, Barone A. A 10-year evaluation of implants placed in fresh extraction sockets: a prospective cohort study. J Periodontol. 2012;83(10):1226-34. https://doi.org/10.1902/ jop.2012.110583.

22. Wu YC, Cheng WC, Chung MP, Su CC, Weng PW, Cathy Tsai YW, et al. Complicated root canal morphology of mandibular lateral incisors is associated with the presence of distolingual root in mandibular first molars: a cone-beam computed tomographic study in a Taiwanese population. J Endod. 2018;44(1):73-9 e1. https://doi.org/10.1016/j.joen.2017.08.027.

23. Kan JY, Roe P, Rungcharassaeng K, Patel RD, Waki T, Lozada JL, et al. Classification of sagittal root position in relation to the anterior maxillary osseous housing for immediate implant placement: a cone beam computed tomography study. Int J Oral Maxillofac Implants. 2011;26(4):8736.

24. Braut V, Bornstein MM, Belser U, Buser D. Thickness of the anterior maxillary facial bone wall-a retrospective radiographic study using cone beam computed tomography. Int J Periodontics Restorative Dent. 2011;31(2):12531.

25. Mandelaris GA, Vence BS, Rosenfeld AL, Forbes DP. A classification system for crestal and radicular dentoalveolar bone phenotypes. Int J Periodontics Restorative Dent. 2013;33(3):289-96. https://doi.org/10.11607/prd.1787.

26. Ghassemian M, Lajolo C, Semeraro V, Giuliani M, Verdugo F, Pirronti T, et al. Relationship between biotype and bone morphology in the lower anterior mandible: an observational study. J Periodontol. 2016;87(6):680-9. https:// doi.org/10.1902/jop.2016.150546.

27. Misch CE. Implant body size: a biomechanical and esthetic rationale. In: Misch CE, editor. Contemporary Implant Dentistry. 3rd ed. St. Louis: Mosby; 2008. p. 160-77.

28. Froum S, Casanova L, Byrne S, Cho SC. Risk assessment before extraction for immediate implant placement in the posterior mandible: a computerized tomographic scan study. J Periodontol. 2011;82(3):395-402. https://doi.org/1 0.1902/jop.2010.100360.

29. Lazzara RJ. Immediate implant placement into extraction sites: surgical and restorative advantages. Int J Periodontics Restor Dent. 1989:9(5):332-43.

30. Zhang S, Shi X, Liu H. Angulations of anterior teeth with reference to the alveolar bone measured by CBCT in a Chinese population. Implant Dent. 2015;24(4):397-401. https://doi.org/10.1097//D.0000000000000291.

31. Araujo $M G$, Lindhe J. Dimensional ridge alterations following tooth extraction. An experimental study in the dog. J Clin Periodontol. 2005;32(2): 212-8. https://doi.org/10.1111/j.1600-051X.2005.00642.x.

32. Cardaropoli G, Araujo M, Hayacibara R, Sukekava F, Lindhe J. Healing of extraction sockets and surgically produced - augmented and nonaugmented - defects in the alveolar ridge. An experimental study in the dog. J Clin Periodontol. 2005;32(5):435-40. https://doi.org/10.1111/j.1600-051 X.2005.00692.x.

33. Grunder U, Gracis S, Capelli M. Influence of the 3-D bone-to-implant relationship on esthetics. Int J Periodontics Restorative Dent. 2005;25(2):1139.

34. Paul S, Held U. Immediate supracrestal implant placement with immediate temporization in the anterior dentition: a retrospective study of 31 implants in 26 patients with up to 5.5-years follow-up. Clin Oral Implants Res. 2013; 24(6):710-7. https://doi.org/10.1111/j.1600-0501.2012.02465.x.

35. Bover-Ramos F, Vina-Almunia J, Cervera-Ballester J, Penarrocha-Diago M, Garcia-Mira B. Accuracy of implant placement with computer-guided surgery: a systematic review and meta-analysis comparing cadaver, clinical, and in vitro studies. Int J Oral Maxillofac Implants. 2018;33(1):101-15. https:// doi.org/10.11607/jomi.5556.

36. Deeb G, Antonos L, Tack S, Carrico C, Laskin D, Deeb JG. Is cone-beam computed tomography always necessary for dental implant placement? J Oral Maxillofac Surg. 2017;75(2):285-9. https://doi.org/10.1016/j.joms.2016.11. 005.

37. Zhang X, Li Y, Ge Z, Zhao H, Miao L, Pan Y. The dimension and morphology of alveolar bone at maxillary anterior teeth in periodontitis: a retrospective 
analysis-using CBCT. Int J Oral Sci. 2020;12(1):4. https://doi.org/10.1038/s413 68-019-0071-0.

38. Van der Weijden F, Dell'Acqua F, Slot DE. Alveolar bone dimensional changes of post-extraction sockets in humans: a systematic review. J Clin Periodontol. 2009;36(12):1048-58. https://doi.org/10.1111/j.1600-051X.2009. 01482.x.

39. Timock AM, Cook V, McDonald T, Leo MC, Crowe J, Benninger BL, et al. Accuracy and reliability of buccal bone height and thickness measurements from cone-beam computed tomography imaging. Am J Orthod Dentofacial Orthop. 2011;140(5):734-44. https://doi.org/10.1016/j.ajodo.2011.06.021.

40. Ahlowalia MS, Patel S, Anwar HM, Cama G, Austin RS, Wilson R, et al. Accuracy of CBCT for volumetric measurement of simulated periapical lesions. Int Endod J. 2013;46(6):538-46. https://doi.org/10.1111/iej.12023.

\section{Publisher's Note}

Springer Nature remains neutral with regard to jurisdictional claims in published maps and institutional affiliations.

\section{Submit your manuscript to a SpringerOpen ${ }^{\circ}$ journal and benefit from:}

- Convenient online submission

- Rigorous peer review

- Open access: articles freely available online

High visibility within the field

- Retaining the copyright to your article

Submit your next manuscript at $\boldsymbol{\nabla}$ springeropen.com 